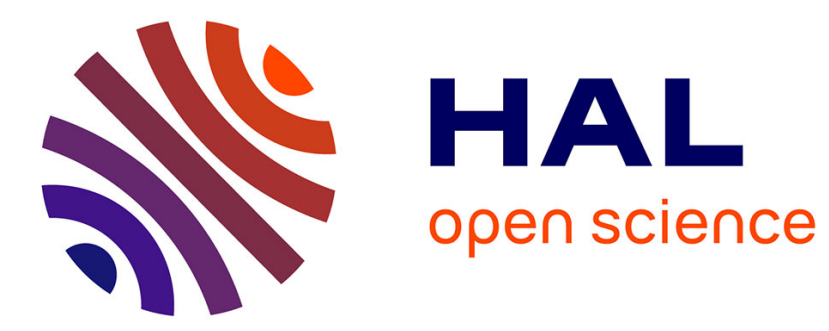

\title{
Digital image correlation used for mechanical tests on crimped glass wool samples
}

Sandra Bergonnier, François Hild, Stéphane Roux

\section{To cite this version:}

Sandra Bergonnier, François Hild, Stéphane Roux. Digital image correlation used for mechanical tests on crimped glass wool samples. Journal of Strain Analysis, 2005, 40 (2), pp.185-197. 10.1243/030932405X7773 . hal-00013799

\section{HAL Id: hal-00013799 https://hal.science/hal-00013799}

Submitted on 17 Dec 2007

HAL is a multi-disciplinary open access archive for the deposit and dissemination of scientific research documents, whether they are published or not. The documents may come from teaching and research institutions in France or abroad, or from public or private research centers.
L'archive ouverte pluridisciplinaire HAL, est destinée au dépôt et à la diffusion de documents scientifiques de niveau recherche, publiés ou non, émanant des établissements d'enseignement et de recherche français ou étrangers, des laboratoires publics ou privés. 


\title{
Digital image correlation used for mechanical tests on crimped glass wool samples
}

\author{
Sandra Bergonnier, ${ }^{1,2}$ François Hild ${ }^{1, *}$ and Stéphane Roux ${ }^{2}$
}

September 8, 2004

\author{
${ }^{1}$ LMT-Cachan, ENS de Cachan / CNRS-UMR 8535 / Université Paris 6 \\ 61 avenue du Président Wilson, F-94235 Cachan Cedex, France \\ Email: sandra.bergonnier@1mt.ens-cachan.fr, francois.hild@1mt.ens-cachan.fr \\ ${ }^{2}$ Laboratoire "Surface du Verre et Interfaces", UMR CNRS/Saint-Gobain \\ 39 quai Lucien Lefranc, F-93303 Aubervilliers Cedex, France \\ Email: stephane.roux@saint-gobain.com
}

\begin{abstract}
Mechanical compression and tearing tests are carried out on crimped glass wool samples. The displacement field is determined by using digital image correlation based on images taken at different stages of the mechanical tests. A multiscale algorithm is used to resolve accurately fine details of the displacement field. This technique reveals strain heterogeneities and further localisation in compression tests well below the peak stress. Crack formations are identified in tearing tests. Reliability and resolution of the displacement and strain fields are validated by using different window sizes in the correlation analysis.
\end{abstract}

Keywords: cracking, large strains, resolution, soft materials, strain localisation.

* corresponding author, Fax: +33147402240 


\section{Notations}

$\boldsymbol{a}$ : in-plane displacement vector

$b$ : noise

$f:$ signal

$g$ : shifted signal

$h$ : cross-correlation

$n$ : integer

$\boldsymbol{u}$ : in-plane displacement vector

$u_{1}$ : local displacement component along the 1-direction

$\boldsymbol{x}:$ position vector

$\boldsymbol{x}_{n}$ : position vector associated to scale no. $n$

A: in-plane strain tensor

$P, Q$ : integers

W: windowing matrix

ZOI: gray level matrix of the zone of interest

$\alpha$ : constant

$\beta$ : constant

$\delta$ : shift between two neighbouring ZOIs

$\delta \boldsymbol{v}$ : correction of in-plane trial displacement vector

$\delta \boldsymbol{\omega}$ : sub-pixel correction of in-plane trial displacement vector

$\bar{\epsilon}$ : in-plane global strain

$\epsilon_{11}$ : local normal strain along the 1-direction

$\sigma_{U}:$ displacement resolution

$\sigma_{\epsilon}:$ strain resolution

$v$ : in-plane trial displacement vector

$\boldsymbol{v}_{0}$ : initial evaluation of in-plane trial displacement vector 
$\boldsymbol{v}_{n}$ : interpolation at scale $n$ of the in-plane displacement field

$\star$ : cross-correlation operator

$\because \therefore$ dummy variable

\section{Introduction}

To model the mechanical properties of materials, one needs constitutive equations validated thanks to mechanical tests. To carry out tests, different measurement techniques can be used, namely, acoustic emission location [1] that corresponds to microcracking events in brittle or quasi-brittle materials such as concrete, composites or rocks, replica techniques [2] that enable one to identify fatigue damage at the sample face, or optical methods. Since optical methods are non-contact measurements [3], they have a large field of application in materials and structural mechanics [4]. Because of the size of the analysed region, the measured displacement fields can reveal heterogeneities at the surface of the sample during mechanical tests. These heterogeneities can be the onset of strain localisation. Cracks can also be analysed by these types of techniques [5, 6]. As optical methods provide a local evaluation of kinematic fields, local strains and/or displacements can be followed during the test and then strain localisation can be identified at a very early stage [7].

The aim of the present paper is to analyse mechanical tests on very soft materials such as mineral wool. So-called compression and tearing tests are two means for assessing the mechanical properties of this class of materials $[\mathbf{8 , 9}$. During these tests, it will be shown that either strain localisation or cracking occur, so that full-field measurement techniques are needed. Among many optical techniques $[10,11,12,13]$, digital image correlation (DIC) $[14,15,16]$ is chosen in the present case. The DIC technique is particularly suited for the analysis of "soft" solids where strain gauges can be difficult to position or may disturb the response of the material (e.g., polymers $[\mathbf{1 7}, \mathbf{1 8}]$, mineral wool [19, 20], wood and paper [21]). Furthermore, the strain levels are greater than a few percents, thereby preventing the use of some of the above-mentioned optical techniques. 
DIC methods are easy to use and have proven to be an efficient tool whose resolution can be extended much below the pixel value (i.e., $10^{-2}$ pixel or below in some cases [22]). When the displacement between two images used for the calculation is too important, classical methods fail to give an accurate result. In that case, a multiscale approach of DIC [20] can be used to allow for large displacements between images with both a very good robustness and resolution. Section 2 introduces the crimping process of mineral wool. The aim of this industrial process is to get better mechanical performances for a given density of the material. Section 3 deals with two DIC methods. The conventional approach and the multiscale algorithm are discussed. A resolution analysis is performed to choose the relevant correlation parameters. Last, the mechanical tests carried out on crimped glass wool samples are analysed in Section 4. Compression tests are performed up to $-20 \%$ global strain. Tearing tests are also carried out on the same kind of samples up to $15 \%$ global strain.

\section{Crimped glass wool}

Mineral wool is a product made of fibres, whose average diameter is micrometric and length millito centimetric. Entangled fibres are bound with a formo-phenolic resin. As produced, the wool has a laminated structure with clearly visible density differences through the product thickness. As such, the compression and tearing resistances of the wool are poor even at medium densities. To increase the mechanical performances, "crimping" is performed. This consists in imposing an on-line compression along the stratification plane to rotate the anisotropy axis along the thickness axis or to buckle these dense planes for a more isotropic texture (Fig. 1). In both cases, for a given density, much better mechanical performances are achieved. Compressive strength can be enhanced by a factor of two or more by crimping. This underlines the key role of the product texture for its mechanical behaviour, and hence a proper characterisation of both the local texture, and the local strain (and their interdependence), is required to optimise the product. This motivates the study detailed in Section 4. 


\section{Displacement measurement by digital image correlation}

DIC is based on the use of an image matching algorithm (i.e., correlation product), which can be

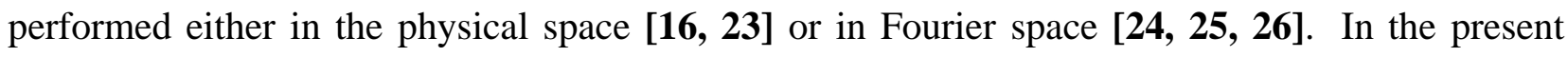
case, Fourier computations are used. The in-plane displacement field is evaluated with the crosscorrelation of an interrogation window in the picture of the deformed surface with respect to a reference one. One advantage of such a method comes from the multipurpose equipment necessary to its practical use, namely: a CCD camera, a fast data acquisition card, a computer and a white light source.

To determine the displacement field of one image of the deformed surface with respect to a reference image, one considers a sub-image which will be referred to as a Zone Of Interest (ZOI). The aim of the correlation method is to match the ZOIs in the two images (Fig. 2). The displacement of a ZOI with respect to the other one is a two-dimensional shift of an intensity signal digitised by a CCD camera. One considers signals $g$ that are merely perturbations of a shifted copy of some reference signal $f$

$$
g(\boldsymbol{x})=f(\boldsymbol{x}-\boldsymbol{u})+b(\boldsymbol{x})
$$

where $\boldsymbol{u}$ is an unknown in-plane displacement vector assumed to be constant locally and $b$ a random noise. To evaluate the displacement $\boldsymbol{u}$, one may minimise the norm of the difference between $f(\boldsymbol{x}-\boldsymbol{v})$ and $g(\boldsymbol{x})$ with respect to a trial displacement $\boldsymbol{v}$

$$
\min _{\boldsymbol{v}}\left(\|g(.)-f(.-\boldsymbol{v})\|^{2}\right)
$$

If one chooses the usual quadratic norm $\|f\|^{2}=\iint|f(\boldsymbol{x})|^{2} \mathrm{~d} \boldsymbol{x}$, the previous minimisation problem is equivalent to maximizing the quantity $h(v)$

$$
h(\boldsymbol{v})=(g \star f)(\boldsymbol{v}) \equiv \iint g(\boldsymbol{x}) f(\boldsymbol{x}-\boldsymbol{v}) \mathrm{d} \boldsymbol{x}
$$

Furthermore, when $b$ is a white noise, the previous estimate is optimal. 


\subsection{Conventional Approach}

In practice, two images are considered. The first one, referred to as "reference image" and the second one, called "deformed image". The reference image, schematically shown in Fig. 3, is meshed by ZOIs that form the region of interest (ROI). The sub-ROI of area $2^{P} \times 2^{Q}$ pixels, is extracted from the ROI in the reference image with the largest values of $P$ and $Q$. A first FFT correlation is carried out on the sub-ROI. The maximum value of the cross-correlation product enables one to estimate the average displacement $\boldsymbol{v}_{0}$. The ROI in the deformed image is then centred at a point corresponding to the centre of the ROI in the reference image moved by an amount $\boldsymbol{v}_{0}$. A second analysis is then performed for each ZOI independently. Each maximum of the calculated crosscorrelation function gives a first value of the in-plane displacement correction $\delta \boldsymbol{v}$. The centres of the ZOI in the deformed image are then moved by an additional amount $\delta \boldsymbol{v}$.

A sub-pixel iterative scheme can be used to have estimations for which the resolution is of the order of a hundredth of pixel for 8-bit pictures [27]. An additional cross-correlation is performed. A subpixel correction of the displacement $\delta \boldsymbol{\omega}$ is obtained by determining the maximum of a parabolic interpolation of the correlation function. The interpolation is performed by considering the maximum pixel and its eight nearest neighbours. Therefore, one obtains a sub-pixel value. By using the "shift-modulation" property of Fourier transform, the deformed ZOI can be moved by an amount $\boldsymbol{\delta} \boldsymbol{\omega}$. Since an interpolation is used, one may induce some (small) errors requiring to re-iterate by considering the new "deformed" ZOI until convergence. The procedure used checks whether the maximum of the interpolated correlation function increases as the number of iterations increases. Otherwise, the iteration scheme is stopped. To limit the influence of edge effects, a windowing of the ZOI can be performed. It consists in constructing a periodic ZOI, $\widehat{\mathrm{ZOI}}$, by averaging the pixels on the edges of the initial ZOI (i.e., "blurring" the edges)

$$
\widehat{\mathrm{ZOI}}=\mathrm{W} \text { ZOI W }
$$

where the matrix $\mathbf{W}$ is equal the identity matrix apart from the four "corners", which are equal to $1 / 2$. 
This first approach is well-suited for small perturbations, but if displacements are too large, the information in the ZOI of the reference image will be partially lost in the ZOI of the deformed image, i.e., there is not enough information to determine a reliable correction $\boldsymbol{\delta} \boldsymbol{v}$. To use the method in the domain of large strains, as for the case of mineral wool, a large ZOI size should be used, whose main drawback is to average displacements over a larger surface. However, the aim of a correlation calculation is to determine displacement fields at the scale of texture heterogeneities. The maximum measurable displacement for the algorithm to converge to the proper solution has then to be increased. This is achieved by using a multiscale algorithm.

\subsection{Multiscale algorithm}

The aim of the multiscale algorithm is to improve the spatial resolution (i.e., to decrease the ZOI size), yet allowing for a large displacement between two consecutive images. The previous approach is recursively applied on images at different scales. Figure 4 shows the construction of images at different scales. Scale no. 0 corresponds to the considered image and scale no. 1 to the region of interest. Scale no. 2, extracted from the ROI, is determined by the largest values for $P$ and $Q$ so that the corresponding area of the sub-ROI is $2^{P} \times 2^{Q}$ pixels (Fig. 3). From scale no. 3 on, each transition is characterised by the definition of super-pixels. The latter are defined recursively from one scale to the next by averaging the gray levels of $2 \times 2$ neighbouring pixels. This procedure is carried out until the minimum size of the sub-image is equal to 128 pixels. The analysis starts at, say, scale no. 4 and ends at scale no. 1 . This allows one to obtain a maximum measurable strain four times larger even if the maximum measurable displacement is still limited [20], as in the standard approach, only to the coarser scale (here scale no. 4).

Starting on the highest (i.e., coarser) scale, for which the sub-image size is at least equal to 128 super-pixels (see Fig. 4), a first correlation is performed. From this first computation, the displacement field $\boldsymbol{v}_{n}$ is interpolated as

$$
\boldsymbol{v}_{n}\left(\boldsymbol{x}_{n}\right)=\mathbf{A} \boldsymbol{x}_{n}+\boldsymbol{a}
$$


To check this first estimate, the centres of each ROI of the "deformed" image are relocated according to these first estimates. A new correlation is performed and corrections to the previous displacement field are evaluated. The iterations are stopped as soon as there is no new correction between two iterations for any ZOI analysed. This iterative procedure was implemented to make the displacement evaluation more robust. This robustness is a key to the success of the procedure. If ever the displacement is not properly evaluated on higher scales, there is no chance of getting a good final result [20]. Consequently, the first evaluations have to be performed very carefully.

When the iterative procedure stops on scale no. $n$, it proceeds to scale no. $(n-1)$. Having identified a first estimate of the displacement field, it is used to position the ZOIs on scale no. $(n-1)$, according to the transformation rules associated to each scale transition. The same iterative scheme is followed for scales no. $(n-1)$ to 2 . In practice, the user can choose between a first evaluation that is very fast since it remains on the pixel (or super-pixel) level, and an accurate evaluation requiring several iterations. On scale no. 1, the user selects either a pixel or a sub-pixel resolution of the displacement by following the above described sub-pixel procedure, thereby allowing for the measurement of small levels of strains as well as large levels that will be evaluated in the following section.

When a sequence of more than two images is analysed, two routes can be followed. The first one consists in considering the same reference image. It follows that the errors are not cumulated but there exists a maximum strain level above which the method fails. The second one considers that the reference image is the deformed image of the previous step (i.e., updating procedure). Under these hypotheses, there is no real limitation, apart from the fact that the errors are now cumulated. Strains of the order of 1 and more are routinely observed in a full-field assessment even with a conventional DIC technique [18]. The procedure, CORRELI ${ }^{\mathrm{LMT}}$, is implemented in MATLAB ${ }^{\mathrm{TM}}$ [28]. On artificially deformed images, strains of the order of $\pm 30 \%$ can be measured $[20,29]$ between two pictures. 


\subsection{Performance of the algorithm}

The aim of this part is to estimate the resolution of the correlation algorithm used herein. Two kinematic quantities are going to be analysed, namely displacements and strains, the resolution of which will be estimated when the correlation parameters are modified. To have independent estimates of the displacements in the following analyses, the shift $\delta$ is equal to the ZOI size (i.e., $\delta=2^{n}$ pixels).

First, the displacement resolution is estimated. In the present case, a constant displacement varying between 0 and 1 pixel, with an increment of 0.1 pixel is applied artificially by using the shift/modulation property of Fourier transforms [27]. The average gives an evaluation of the error and the standard deviation that of the corresponding resolution. These properties are associated to the analysed texture. Figure 5 shows the average error and the corresponding standard deviation as a function of the applied displacement for different ZOI sizes. For a given ZOI size, the maximum error is reached when the prescribed displacement is equal to 0.5 pixel. In this case, the information between each ZOI in the reference and deformed pictures is the most biased. Furthermore, the larger the ZOI, the smaller the error. However, if the ZOI size increases, the spatial resolution is degraded, i.e., less independent measurement points are obtained. Second, the strain resolution is determined by using the same sequence of pictures. From the displacement field, the strains are evaluated by using a classical finite differences scheme so that the spatial resolution (i.e., the gauge length) is equal to $2 \times \delta$, i.e., $2^{n+1}$ pixels. In all cases, the mean strain is less than $4 \times 10^{-5}$. The strain resolution is more sensitive to $\delta$ (here equal to the ZOI size) than the displacement resolution to the ZOI size (Fig. 6-a).

Figure 6-b shows the change of the mean displacement and strain resolutions with the ZOI size when the displacement range varies between 0 and 1 pixel. For $n \geq 4$ a power law with an exponent of the order -1 is obtained for the mean displacement resolution $\sigma_{U}$

$$
\sigma_{U}=\frac{\alpha^{2}}{2^{n}}
$$

with $\alpha=0.64$ pixel, thereby indicating that for a large range of ZOI sizes, the displacement reso- 
lution and the spatial resolution are the result of a compromise described by Eqn. (6). Let us note that Eqn. (6) shows a non trivial dependence. Such a formula can be understood in the context of the central limit theorem, when deviations from a uniform translation can be described as a white noise with a correlation length smaller that the ZOI size. In that case, the resolution varies as the inverse square root of the number of pixels in the ZOI (i.e., $1 / \sqrt{2^{n} \times 2^{n}}$ ). This result will be wrong as soon as long range correlations (e.g., power-law decay) will exist at large scales. Moreover, the mean strain resolution is inversely proportional to $\delta$ (here equal to the ZOI size) so that

$$
\sigma_{\epsilon}=\beta \frac{\sigma_{U}}{2 \delta}
$$

with $\beta=1.56$, so that an increase of the "gauge length" $2 \delta$ yields a better strain resolution, yet a poorer spatial resolution. Equation (7) is in agreement with the results shown in Fig. 6-b since a power law with an exponent -2 is found when the shift parameter $\delta$ is equal to the ZOI size. The origin of such a dependence can be understood by the fact that the finite difference operation relates the resolution of the displacement $\sigma_{U}$ and that of the strain $\sigma_{\epsilon}$ as proportional to each other, and inversely proportional to $2 \delta$. Let us note that this equation also survives in the case of long range correlations (with a different prefactor $\beta$ ). Consequently, displacement and strain resolutions are simply related in all cases by Eqn. (7).

Let us note that the displacement resolution is analogous to a frequency (or spectral) resolution, namely, the highest (or Nyquist) frequency controls the displacement resolution. To improve the resolution in displacements or strains, one has to increase the ZOI size as much as possible. Simultaneously, since averages are taken over larger and larger zones, all information on spatial localization is lost. For the latter, the natural "spatial resolution" is simply given by the ZOI size. Such a competition between spatial and spectral resolution (where the latter controls, in the present case, the strain resolution) is a ubiquitous property at play in a wide variety of different contexts, from Heisenberg's principle in quantum mechanics to signal processing [30], where this question is at the very heart of the invention of wavelets [31]. Here a similar limitation is encountered in the quest for both a spatially resolved and an accurate kinematic measurement. 
A good compromise for the present study is given by a ZOI size of 32 pixels (i.e., $n=5$ ) for which an average displacement resolution less than 0.015 pixel is achieved. In terms of strains, the corresponding average resolution is less than $4 \times 10^{-4}$ with a spatial resolution of 64 pixels. It should be remembered that all the results discussed so far were obtained with the same reference picture. When large strains occur or severe texture variations [20], an updating procedure is preferred. Consequently the resolution gradually degrades as the number of analysed pictures increases.

With the chosen correlation parameters, two cases are now studied. An average strain of $2 \%$ and $20 \%$ is applied by using a linear interpolation of each gray level to construct the artificially deformed images. To determine the average strain, the displacement field is linearly interpolated and the slope corresponds to the estimated average strain. From this interpolation, the displacement residuals are obtained as the difference between the measured displacement and the interpolation. The corresponding strain resolution is estimated by the standard deviation of strain associated to the residual displacement field. For any correlation parameter $3 \leq n \leq 7$, there is a difference of $3 \times 10^{-4}$ at most between the measured mean strain and its prescribed value (i.e., $2 \times 10^{-2}$ ). When $n=5$, the corresponding resolution is less than $3 \times 10^{-3}$. For a prescribed strain of $2 \times 10^{-1}$, the difference between the mean measured strain and the prescribed value is less than $10^{-3}$ for all the correlation parameters. The strain resolution is of the order of $10^{-2}$ when $n=5$. In the following section, this multiscale technique is applied to the analysis of mechanical tests on crimped glass wool samples with $n=5$.

\section{Experiments: mechanical tests on crimped glass wool}

To assess the mechanical properties of crimped glass wool, mechanical tests are carried out. All the tests reported herein are displacement-controlled (or equivalently, the global strain $\bar{\epsilon}$ is controlled; it is defined as the ratio of the displacement between the two platens divided by the initial height of the sample). A uniaxial compression (in the 1-direction) up to $-20 \%$ global strain, is applied on 
parallelepipedic samples, whose dimensions are $200 \times 100 \times 80 \mathrm{~mm}^{3}, 80 \mathrm{~mm}$ being the height of the sample and tearing tests (in the 1-direction) up to approximately $15 \%$ global strain, applied on parallelepipedic samples, whose dimensions are $200 \times 200 \times 80 \mathrm{~mm}^{3}$. For the tests, images of the sample illuminated with direct light have been taken for different global strain levels (with $-0.5 \%$ increments in compression and $+0.5 \%$ in tearing). In the present case, no special preparation was needed since the natural (random) texture of the studied material was sufficient to determine displacement fields and subsequently strain fields.

For the compression tests, the main information is given by the local strain to be compared to the global level. The strain distribution will be analysed with respect to the overall force vs. global strain. In tearing tests, macroscopic cracks initiate and propagate. Consequently, the relevant quantity to study is the displacement field so that the above-mentioned stages of cracking can be monitored. In all the experiments reported herein, two types of fields are shown. First, the so-called cumulative fields for which the analysed kinematic quantity is evaluated for a reference given prior to the beginning of the experiment. This quantity is generally given to describe an experiment. To study active zones incrementally, the fields are also evaluated between a current state and the previous state for which a picture was taken (i.e., for a global strain increment of $\pm 0.5 \%$ ). In terms of results, the same relative resolution is to be expected for the two types of fields since the same type of texture is considered with the same (i.e., multiscale) procedure.

\subsection{Uniaxial compression tests}

The result of the test is shown in Fig. 7. The "plastic" plateau suggests the existence of localised strains. This implies to study the strain field of the material with a good spatial resolution. In Fig. 8, strain fields are shown. The calculation, which is performed with ZOIs of size 32 pixels with a 32-pixel shift $\delta$, corresponds to the component $\epsilon_{11}$ for different global strain levels, namely, $-4 \%$ for Fig. 8(a), $-6.5 \%$ for Fig. 8(b) and $-9 \%$ for Fig. 8(c), respectively. In Fig. 7, the corresponding points are located at the end of the elastic domain, at the beginning of the "plastic" plateau and 
where the "plastic" plateau is well-defined.

Figures 8 show the change of the strain field during the test. From $-4 \%$ global strain in Fig. 8(a), strain heterogeneities can be identified at the surface of the material. After a global strain level of $-9 \%$, a well-marked localised zone splits into two bands on the left of the sample. More importantly, precursors of this localised band can be seen at earlier stages (Fig. 8(b) clearly, and Fig. 8(a) more faintly). All along the test, the strain fluctuations are located only in the identified bands, and their width remains constant as the test goes on. Furthermore, the incremental strain levels vary within the band during the test. These two phenomena can be observed on the incremental strain maps in Figs. 8. At the end of the test, the localisation band is about $8 \mathrm{~mm}$ wide, i.e., one tenth of the height of the sample.

\subsection{Tearing tests}

The overall result of a tearing test is shown in Fig. 9. The end of the elastic domain corresponds to the inception of cracking. As the test proceeds, another crack appears at the surface of the material, which prevents the opening of the first one because of stress shielding. In Fig. 10, correlation results are shown. The calculation, performed with ZOIs of size 32 pixels with a 32-pixel shift between two ZOIs, corresponds to the vertical displacement field $u_{1}$ for different global strain levels, namely, 4\% for Fig. 10(a), 4.5\% for Fig. 10(b) and 5.5\% for Fig. 10(c), respectively.

In Fig. 10(a), the displacement field $u_{1}$ at the surface of the sample corresponds to the inception of the first crack. At that moment, the displacement field becomes heterogeneous. In Fig. 10(b), discontinuities in the incremental and cumulative vertical displacement field $u_{1}$ can be observed. The discontinuity corresponds to an opening crack. In Fig. 10(c), another crack appears at the surface. This corresponds to the displacement discontinuity that can be observed on the cumulative displacement map. In the incremental displacement, the discontinuity also appears and one can see that the first crack stops opening, being shielded by the second one. At the end of the test, the crack 
completely traverses the sample, and constitutes the final failure mode. This multiscale correlation calculation enables one to reveal displacement discontinuities or cracks, at the surface of the material. As it is a surface measurement, one cannot exclude that cracks may have been initiated earlier in the bulk of the material without intersecting with the analysed face of the sample.

\subsection{Influence of correlation parameters on results}

Correlation calculations are also performed with other parameters (a smaller shift $\delta$ between two consecutive ZOIs) to study their influence on the results. The two tests are analysed again.

For the compression test, one can expect, for instance, that the width of the localisation band will depend on the shift between two consecutive ZOIs. A calculation with a 16-pixel shift $\delta$ has been performed. Figure 11 shows the correlation results for the same global strain as the results presented for a 32-pixel shift in Section 4.1. For such a calculation, for the same ROI, the number of ZOIs in the height of the image is twice as important as for a 32-pixel shift. When the results are compared, one can observe that strain localisation appears for the same image and that the two identified bands are identically positioned. Moreover, in Figs. 11 and 8, the width of the bands are the same whereas the number of ZOIs belonging to the bands are different. If strain levels are compared, the two calculations have the same scales to analyse the results. In the present case, the influence of the correlation parameters is small when the scale of the measurement is relevant with respect to the underlying mechanism (e.g., strain localisation).

For the tearing test, the difference between two calculations with different shifts $\delta$ is greater because cracks correspond to discontinuities in the displacement field. If the crack belongs to only one ZOI, the discontinuity represents the whole crack, but if more than one ZOI makes up the crack, the mouth of the crack might be visible. Figure 12 shows the correlation results for a 16-pixel shift $\delta$. The results for the same global strain rate as those in Figs. 10 are shown. On the incremental field, it can be observed that, for a 16-pixel shift, the displacement field is more heterogeneous at the 
surface of the sample. For a $4.5 \%$ global strain (Fig. 12(b)), the first crack can clearly be identified. The crack mouth appears at the surface of the sample, which cannot be seen for a 32-pixel shift. For the latter calculation, the crack is made up of more than one ZOI, and its opening is visible. In Fig. 12(c), the second crack can be seen. The results of the correlation calculation depend on the parameters. Their influence affects the results, however in a controlled manner. If the method is used to detect the presence of a crack, a coarse calculation, with a shift between two consecutive ZOIs of the same size than the ZOI, is effective enough. If the size of the crack is looked for, a finer calculation is required.

\section{Conclusion}

An original approach of displacement and strain fields identification has been presented. Beyond the conventional DIC technique, which allows for a first evaluation of the displacement field, the image is resized and relocated to a coarser scale to increase both the robustness and the maximum measurable displacement and strain between two consecutive images. A resolution analysis is performed so that the correlation parameters can be chosen. This multiscale approach has then been applied to mechanical tests by using the updating procedure.

First, it is applied to a uniaxial compression test on a crimped glass wool sample. The technique enables one to identify strain heterogeneity zones, and then strain localisation bands. If the correlation parameters are changed, the results are very similar, namely, the strain level is the same, heterogeneities appear for the same global level, and localisation bands have the same width for a given global strain level.

Second, by using the same procedure, tearing tests have been carried out on crimped glass wool. Discontinuities in the displacement field, early onset of tearing or the growth of a crack can be observed. The influence of calculation parameters has been quantified. If only the presence of a crack or a localisation pattern is looked for, a coarse and quick calculation can be performed whereas if 
detailed analysis is to be carried out, a finer scale has to be used.

The correlation technique applied to crimped glass wool shows the change of macrostructure during the tests. As crimped glass wool is a highly textured material, the change in local orientations of constitutive fibers can be characterised to study the link between the texture and strain localisation. A further development is then to relate the obtained displacement and strain fields to local heterogeneities of the crimped glass wool texture and to evaluate the a priori mechanical performances from the texture, and hence optimise crimping.

\section{Acknowledgements}

This work has been supported by Isover Saint-Gobain and by CNRS in a project entitled "Analyses multi-échelles de champs de déformation par traitement d'image : vers l'identification de champs de propriétés mécaniques.” The authors acknowledge useful discussions with O. Gaume, C. Machelart and J.-B. Rieunier. 


\section{References}

[1] Drouillard, T.F. A History of Acoustic Emission. J. Acoust. Em., 1996 14(1), 1-34.

[2] ISO 3057-1974. NDT-Metallographic Replica Techniques of Surface Examinations, 1974.

[3] Rastogi, P.K., Edt. Photomechanics, (Springer, Berlin), 2000.

[4] Jacquot, P. and Fournier, J.-M., Edts. Interferometry in Speckle Light, Theory and Applications, (Springer, Berlin), 2000.

[5] Dawicke, D.S. and Sutton, M.S. CTOA and Crack-Tunneling Measurements in Thin Sheet 2024-T3 Aluminum Alloy. Exp. Mech., 1994 34, 357-368.

[6] Humbert, L., Valle, V. and Cottron, M. Experimental Determination and Empirical Representation of Out-of-Plane Displacements in a Cracked Elastic Plate Loaded in Mode I. Int. J. Solids Struct., 2000 37, 5493-5504.

[7] Desrues, J., Lanier, J. and Stutz, P. Localization of the Deformation in Tests on Sand Samples. Eng. Fract. Mech., 1985 21(4), 251-262.

[8] EN 826. Thermal insulating products for building applications - Determination of compression behaviour. European standard, 1996.

[9] EN 1607. Thermal insulating products for building applications - Determination of tensile strength perpendicular to faces. European standard, 1996.

[10] Coker, E.G. and Filon, L.N.G. A Treatise on Photoelasticity, (Cambridge University Press, New York), 1931.

[11] Guild, J. Diffraction Gratings as Measuring Scales, Practical Guide to Metrological Use of Moiré Fringes, (Oxford University Press, Oxford), 1960.

[12] Smigielski, P. Holographie industrielle, (Teknea, Toulouse), 1994.

[13] Dudderar, T.D. and Simpkins, P.G. Laser Speckle Photography in a Fluid Medium. Nature, $1977270,45-47$. 
[14] Burt, P.J., Yen, C. and Xu, X. Local Correlation Measures for Motion Analysis: a Comparative Study. In Proceedings IEEE Conf. on Pattern Recognition and Image Processing, 1982, pp. 269-274.

[15] Peters, W.H. and Ranson, W.F. Digital Imaging Techniques in Experimental Stress Analysis. Opt. Eng., 1982 21, 427-431.

[16] Sutton, M.A., Wolters, W.J., Peters, W.H., Ranson, W.F. and McNeill, S.R. Determination of Displacements Using an Improved Digital Correlation Method. Im. Vis. Comp., 1983 1(3), 133-139.

[17] G'Sell, C., Hiver, J.-M., Dahnoun, A. and Souahi, A. Video-Controlled Tensile Testing of Polymers and Metals Beyond the Necking Point. J. Mat. Sci., 1992 27, 5031-5039.

[18] Chevalier, L., Calloch, S., Hild, F. and Marco, Y. Digital Image Correlation used to Analyze the Multiaxial Behavior of Rubber-Like Materials. Eur. J. Mech. A/Solids, 2001 20, 169-187.

[19] Roux, S., Hild, F. and Berthaud, Y. Correlation Image Velocimetry: A Spectral Approach. Appl. Optics, 2002 41(1), 108-115.

[20] Hild, F., Raka, B., Baudequin, M., Roux, S. and Cantelaube, F. Multi-Scale Displacement Field Measurements of Compressed Mineral Wool Samples by Digital Image Correlation. Appl. Optics, 2002 IP 41(32), 6815-6828.

[21] Choi, D., Thorpe, J.L. and Hanna, R. Image Analysis to Measure Strain in Wood and Paper. Wood Sci. Technol., 1991 25, 251-262.

[22] Sutton, M. A., McNeill, S. R., Jang J. and Babai M., Effects of subpixel image restoration on digital correlation error estimates, Opt. Eng., 1988 27(10) 870-877.

[23] Chu, T.C., Ranson, W.F., Sutton, M.A. and Petters, W.H. Applications of Digital-ImageCorrelation Techniques to Experimental Mechanics. Exp. Mech., 1985 3(25), 232-244.

[24] Chen, D.J., Chiang, F.P., Tan, Y.S. and Don, H.S. Digital Speckle-Displacement Measurement Using a Complex Spectrum Method. Appl. Opt., 1993 32, 1839-1849. 
[25] Berthaud, Y., Scholz, J. and Thesing, J. Méthodes optiques et acoustiques de mesures des caractéristiques mécaniques. Proceedings Colloque national MECAMAT 'Mécanismes et mécanique des grandes déformations', 1996, pp. 77-80.

[26] Chiang, F.P., Wang, Q. and Lehman, F. New Developments in Full-Field Strain Measurements Using Speckles. In Non-Traditional Methods of Sensing Stress, Strain and Damage in Materials and Structures, (ASTM, Philadelphia), 1997, pp. 156-169.

[27] Périé, J.N., Calloch, S., Cluzel, C. and Hild, F. Analysis of a Multiaxial Test on a C/C Composite by Using Digital Image Correlation and a Damage Model. Exp. Mech., 2002 42(3), 318-328.

[28] Matlab. Matlab 5.3, the Language of Technical Computing, version 5.3, (the MathWorks, inc. (http://www.mathworks.com), 1999).

[29] Chevalier, L. and Marco, Y. Tools for Multiaxial Validation of Behavior Laws Chosen for Modeling Hyper-Elasticity of Rubber-Like Materials. Polym. Eng. Sci., 2002 42(2), 280-298.

[30] Gabor, D. Theory of Communication. J. IEE, 1946 93(3), 429-457.

[31] Mallat, S. A wavelet tour of signal processing, (Academic Press, San Diego), 1998. 


\section{List of Figures}

1 Lateral view of a crimped glass wool product $(1$ pixel $\leftrightarrow 0.16 \mathrm{~mm}) . \quad \ldots \ldots 22$

2 Zone of Interest (ZOI) in reference (left) and "deformed” (right) images. Associated displacement vector. . . . . . . . . . . . . . . . . 23

3 Schematic of the reference image with the correlation parameters (ZOI area: $2^{n} \times$ $2^{n}$ pixels and shift $\left.\delta\right) \ldots \ldots \ldots \ldots \ldots \ldots \ldots \ldots$

4 Different scales used in the multi-scale approach for a glass wool sample. . . . . . 25

5 Mean displacement error (a) and corresponding standard deviation (b) as a function of prescribed constant displacement for different correlation parameters (ZOI size

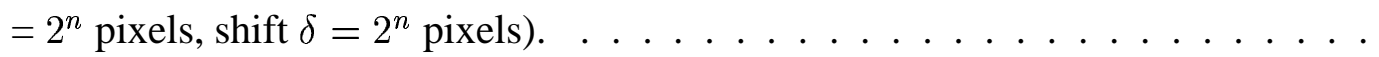

6 -a- Standard deviation of strain as a function of prescribed constant displacement for different correlation parameters. -b- Mean strain and displacement standard deviations as a function of prescribed constant displacement for different correlation parameters $\left(\right.$ ZOI size $=2^{n}$ pixels, shift $\delta=2^{n}$ pixels $) \ldots \ldots \ldots \ldots 27$

7 Dimensionless load vs. global strain for a compression test of crimped glass wool. . 28

8 Cumulative and incremental $\epsilon_{11}$-field for different levels of the global strain in a compression test: $\bar{\epsilon}_{11}=-4 \%$ (a), $\bar{\epsilon}_{11}=-6.5 \%$ (b) and $\bar{\epsilon}_{11}=-9 \%$ (c). Correlation parameters: ZOI size $=32$ pixels, $\delta=32$ pixels. Scale: 1 pixel $\leftrightarrow 0.085 \mathrm{~mm} . \quad \ldots \quad 29$

9 Dimensionless load vs. global strain for a tearing test of crimped glass wool. . . . . 30

10 Cumulative and incremental displacement field $u_{1}$ expressed in pixels for different levels of global strain in a tearing test: $\bar{\epsilon}_{11}=4 \%$ for (a), $\bar{\epsilon}_{11}=4.5 \%$ for (b) and $\bar{\epsilon}_{11}=5.5 \%$ for (c). Correlation parameters: ZOI size $=32$ pixels, $\delta=32$ pixels. Scale: 1 pixel $\leftrightarrow 0.16 \mathrm{~mm} . \ldots \ldots \ldots \ldots \ldots$

11 Cumulative and incremental $\epsilon_{11}$-field for different levels of the global strain in a compression test : $\bar{\epsilon}_{11}=-4 \%$ for (a), $\bar{\epsilon}_{11}=-6.5 \%$ for (b) and $\bar{\epsilon}_{11}=-9 \%$ for (c). Correlation parameters: ZOI size $=32 \times 32$ pixels, $\delta=16$ pixels. Scale: 1 pixel $\leftrightarrow$

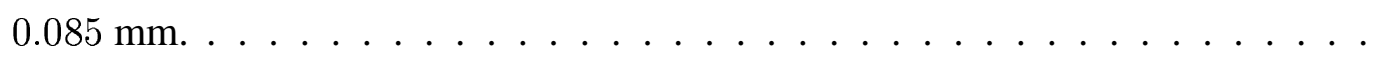


12 Cumulative and incremental displacement field $u_{1}$ expressed in pixels for different levels of global strain in a tearing test: $\bar{\epsilon}_{11}=4 \%$ for (a), $\bar{\epsilon}_{11}=4.5 \%$ for (b) and $\bar{\epsilon}_{11}=5.5 \%$ for (c). Correlation parameters: ZOI size $=32$ pixels, $\delta=16$ pixels. Scale: 1 pixel $\leftrightarrow 0.16 \mathrm{~mm} . \ldots \ldots \ldots \ldots$ 


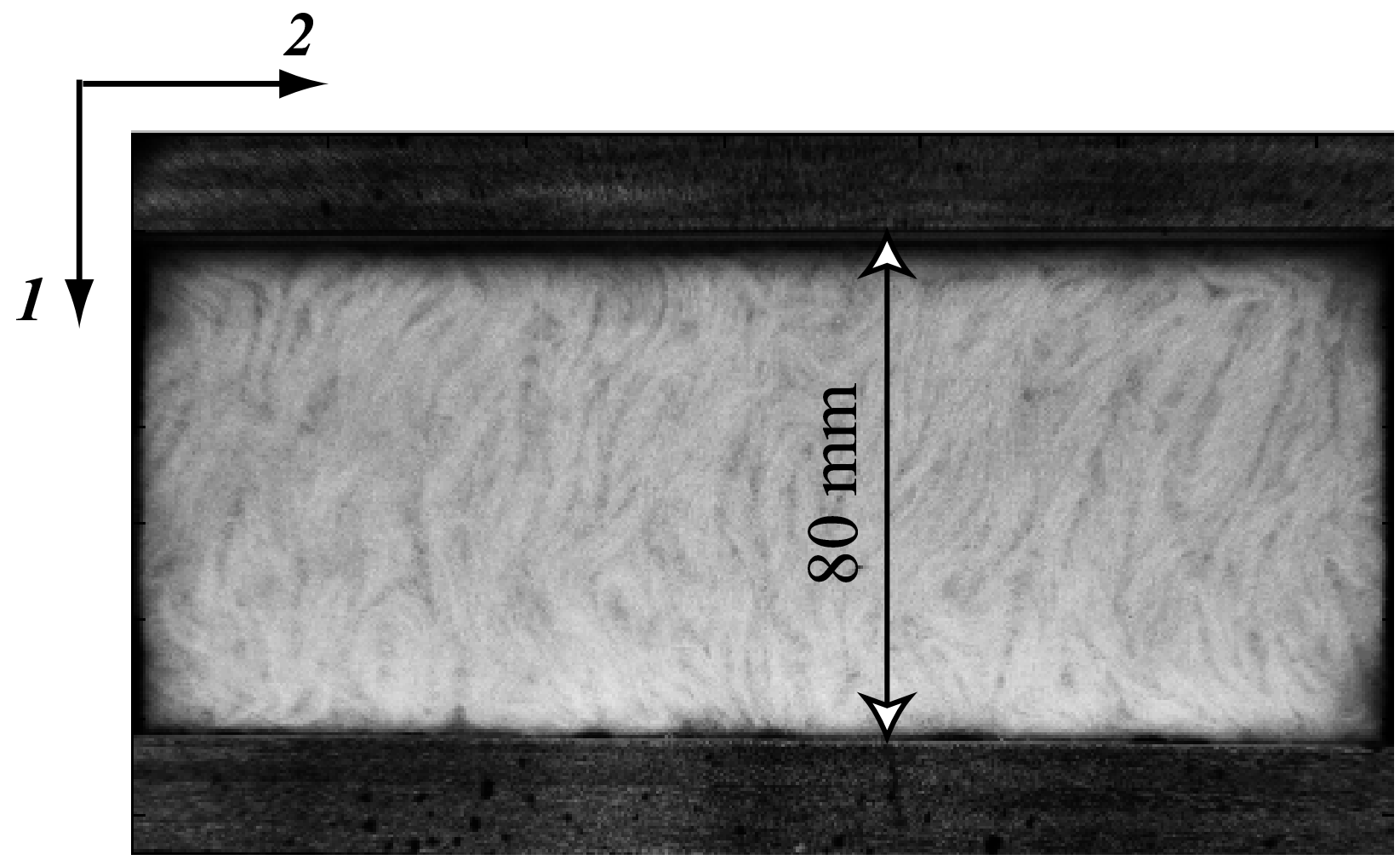

Figure 1: Lateral view of a crimped glass wool product (1 pixel $\leftrightarrow 0.16 \mathrm{~mm}$ ).

Bergonnier et al. 

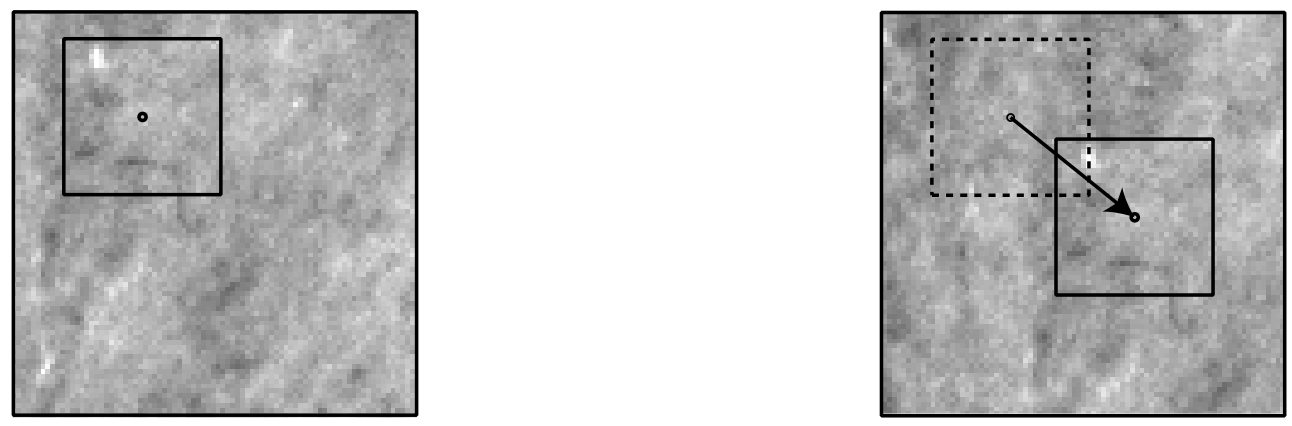

Figure 2: Zone of Interest (ZOI) in reference (left) and "deformed" (right) images. Associated displacement vector.

Bergonnier et al. 


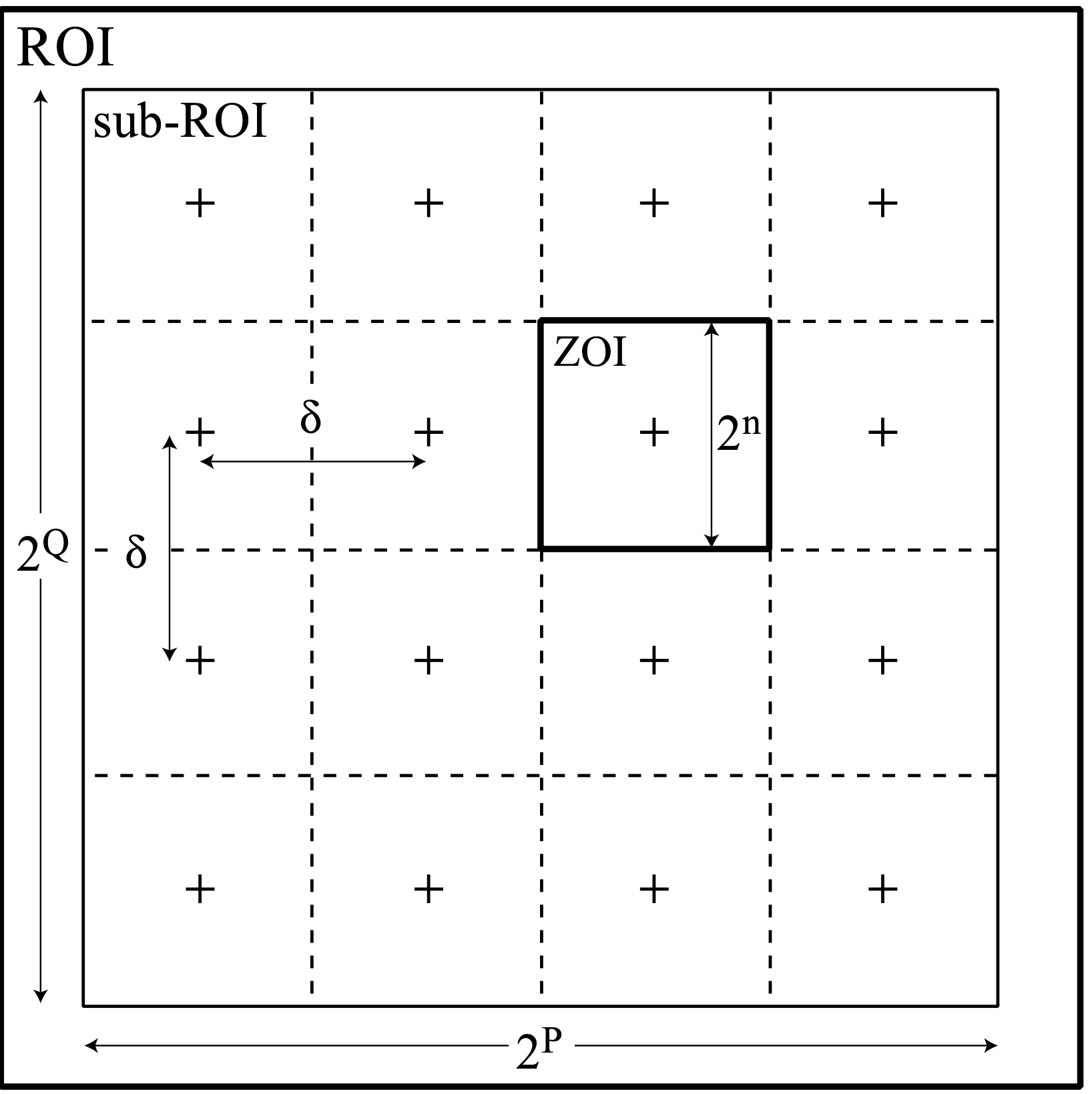

Figure 3: Schematic of the reference image with the correlation parameters (ZOI area: $2^{n} \times 2^{n}$ pixels and shift $\delta$ ).

Bergonnier et al. 


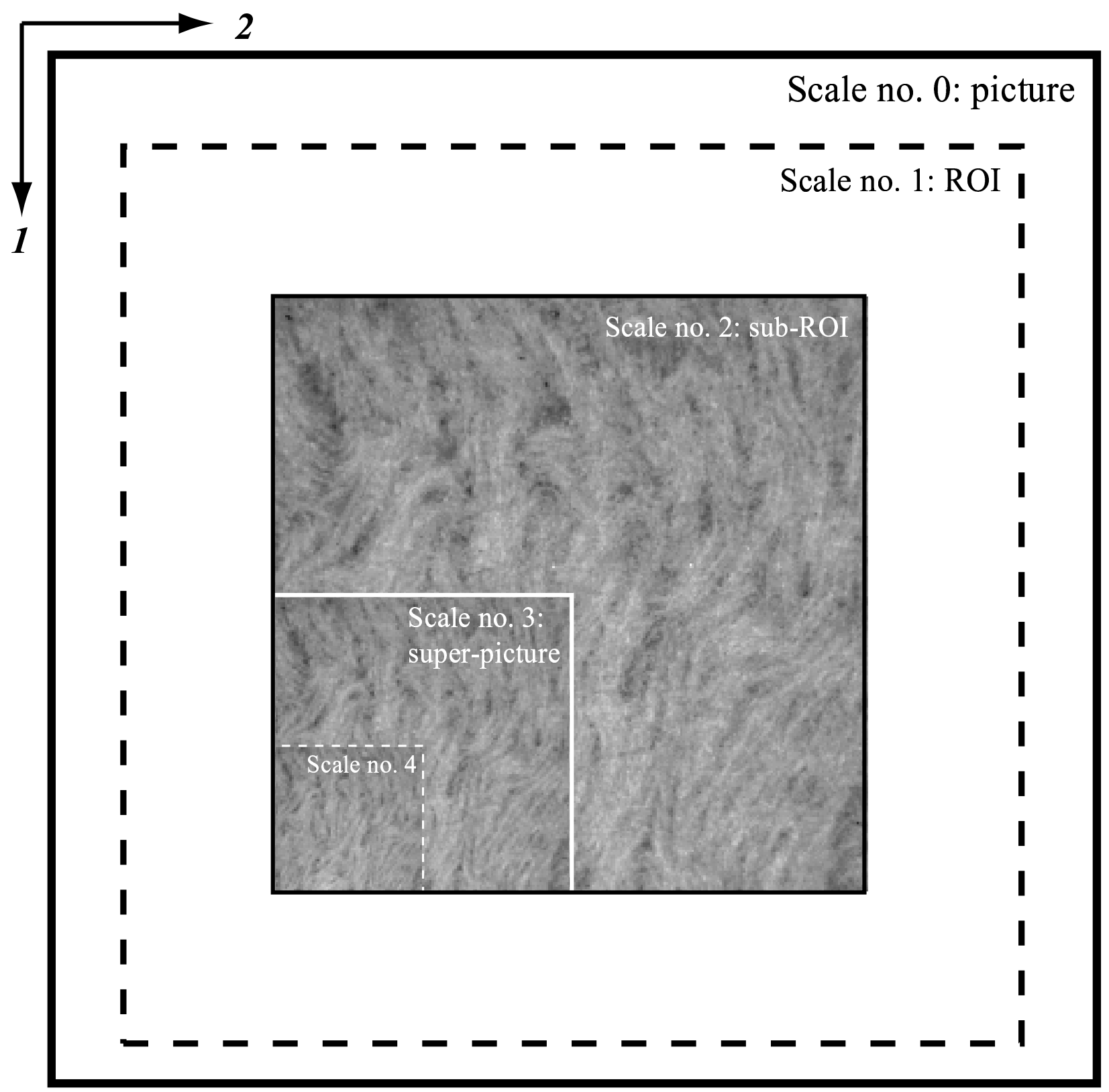

Figure 4: Different scales used in the multi-scale approach for a glass wool sample.

Bergonnier et al. 


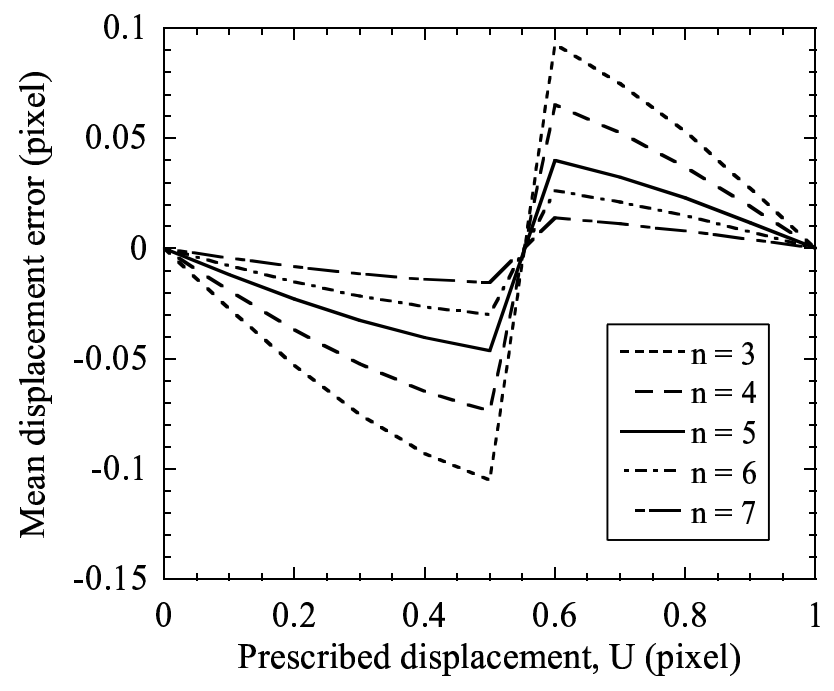

$-\mathrm{a}-$

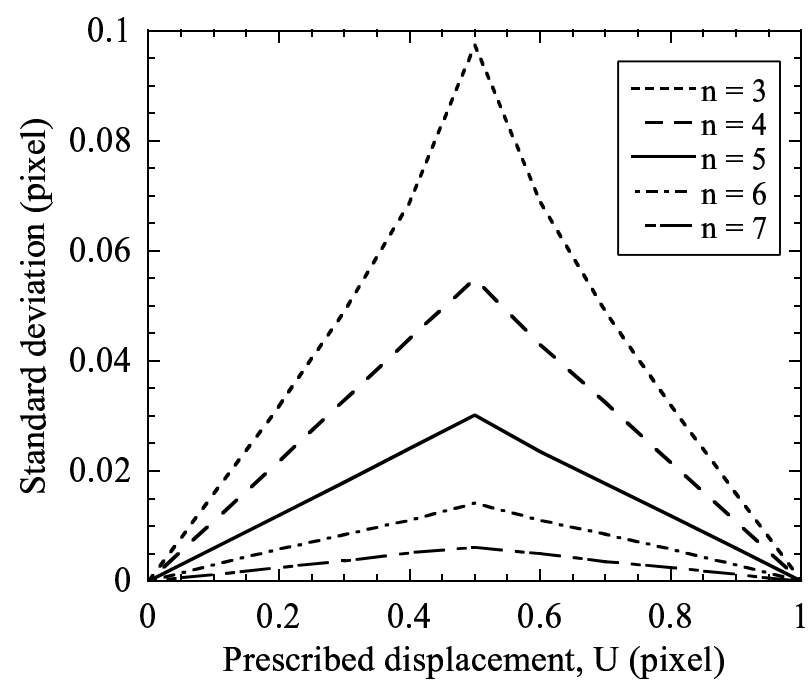

$-b-$

Figure 5: Mean displacement error (a) and corresponding standard deviation (b) as a function of prescribed constant displacement for different correlation parameters (ZOI size $=2^{n}$ pixels, shift $\delta=2^{n}$ pixels).

Bergonnier et al. 


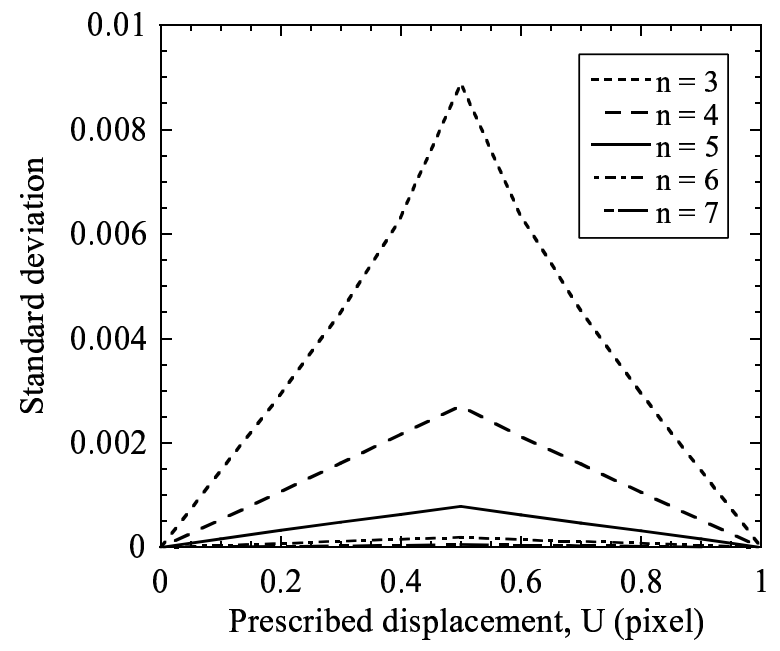

- a -

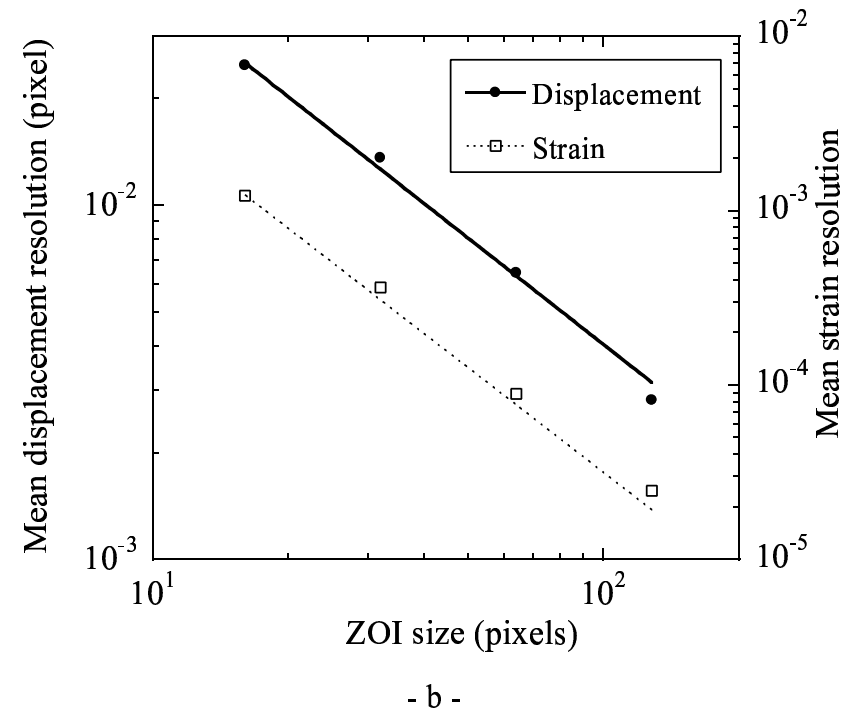

Figure 6: -a- Standard deviation of strain as a function of prescribed constant displacement for different correlation parameters. -b- Mean strain and displacement standard deviations as a function of prescribed constant displacement for different correlation parameters (ZOI size $=2^{n}$ pixels, shift $\delta=2^{n}$ pixels).

Bergonnier et al. 
Fig. 8(c)

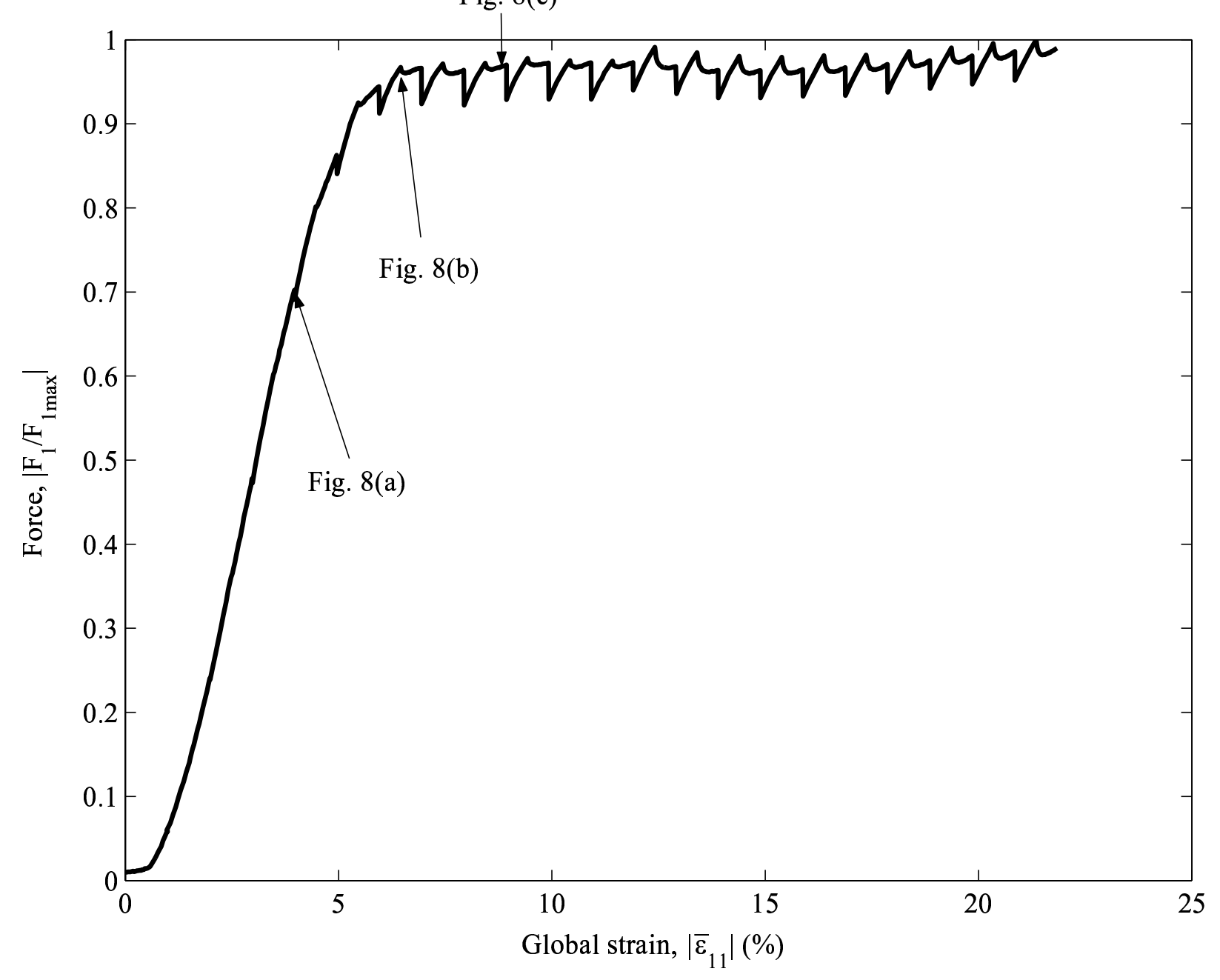

Figure 7: Dimensionless load vs. global strain for a compression test of crimped glass wool.

Bergonnier et al. 

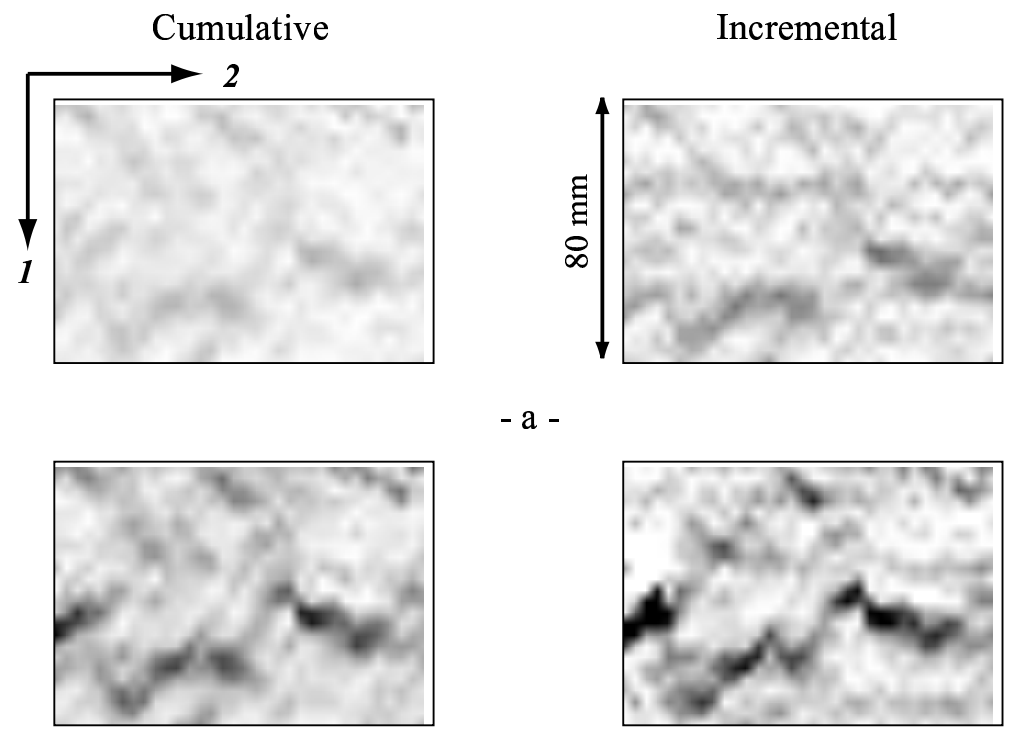

$-b-$
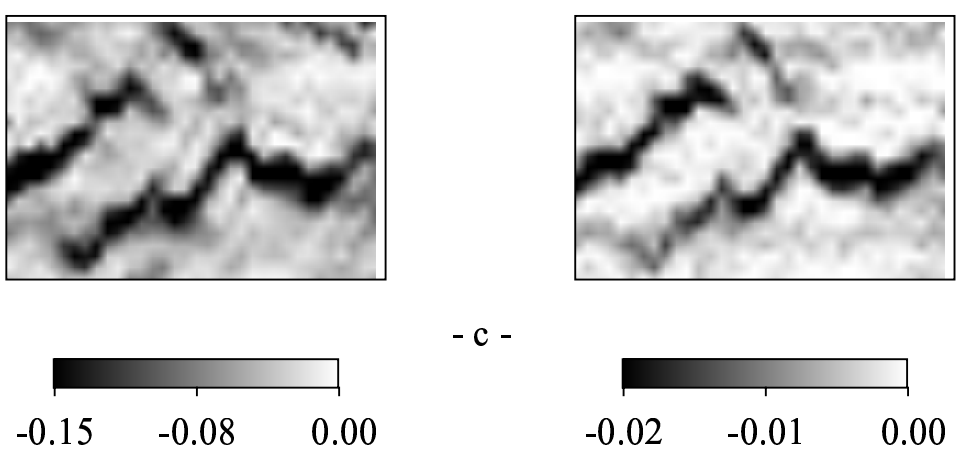

Figure 8: Cumulative and incremental $\epsilon_{11}$-field for different levels of the global strain in a compression test: $\bar{\epsilon}_{11}=-4 \%$ (a), $\bar{\epsilon}_{11}=-6.5 \%$ (b) and $\bar{\epsilon}_{11}=-9 \%$ (c). Correlation parameters: ZOI size $=32$ pixels, $\delta=32$ pixels. Scale: 1 pixel $\leftrightarrow 0.085 \mathrm{~mm}$.

Bergonnier et al. 


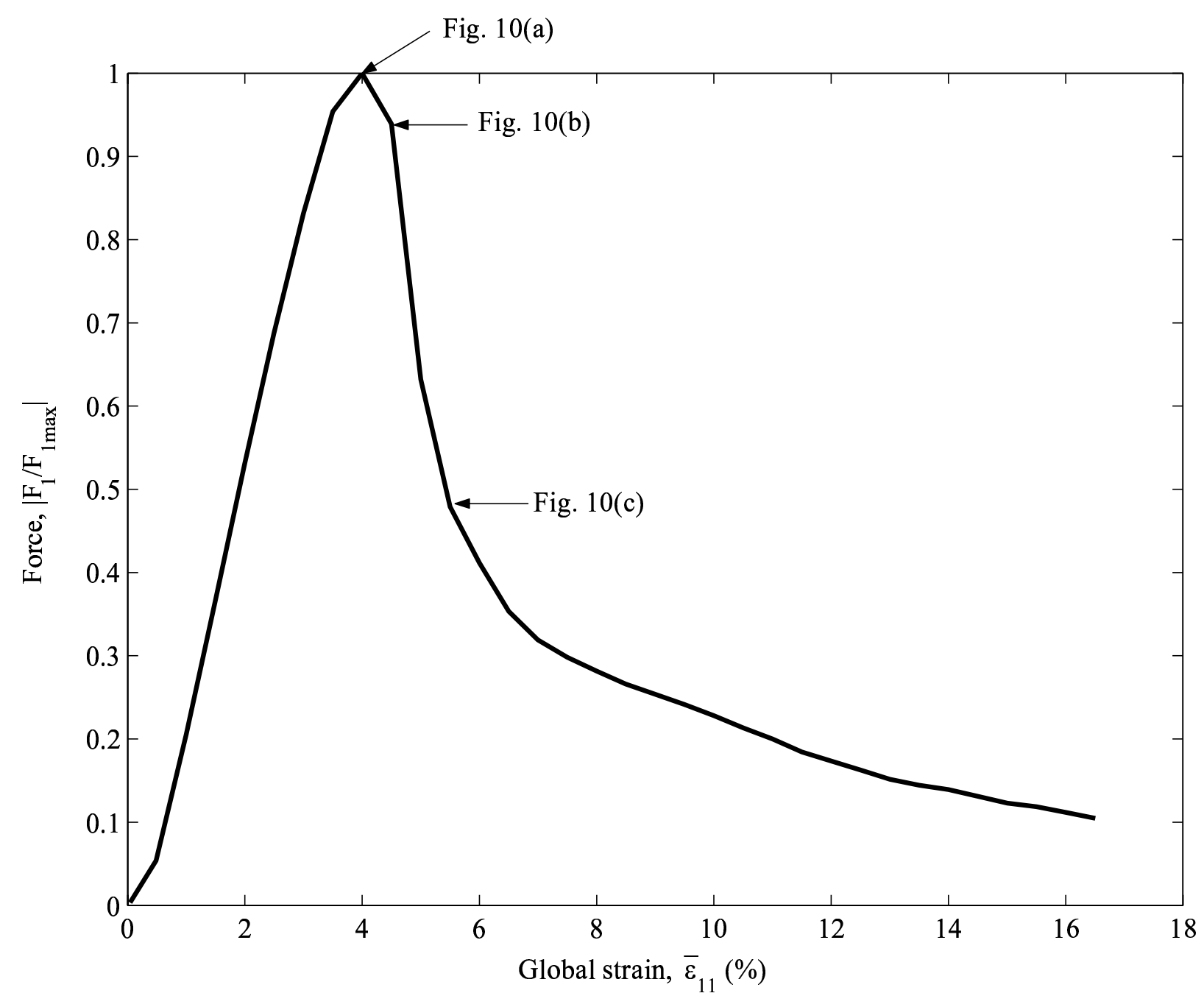

Figure 9: Dimensionless load vs. global strain for a tearing test of crimped glass wool.

Bergonnier et al. 

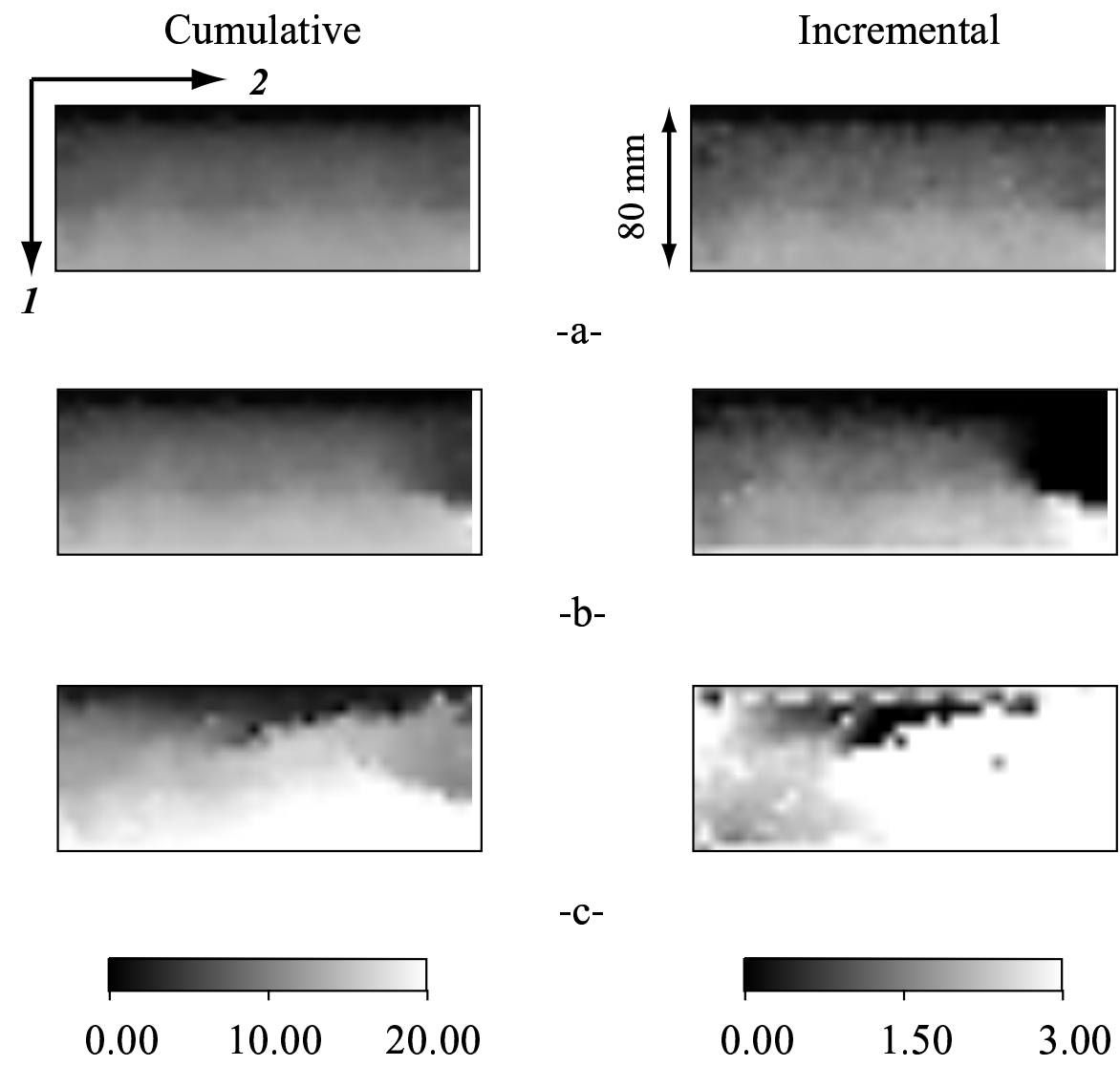

Figure 10: Cumulative and incremental displacement field $u_{1}$ expressed in pixels for different levels of global strain in a tearing test: $\bar{\epsilon}_{11}=4 \%$ for (a), $\bar{\epsilon}_{11}=4.5 \%$ for (b) and $\bar{\epsilon}_{11}=5.5 \%$ for (c). Correlation parameters: ZOI size $=32$ pixels, $\delta=32$ pixels. Scale: 1 pixel $\leftrightarrow 0.16 \mathrm{~mm}$.

\section{Bergonnier et al.}



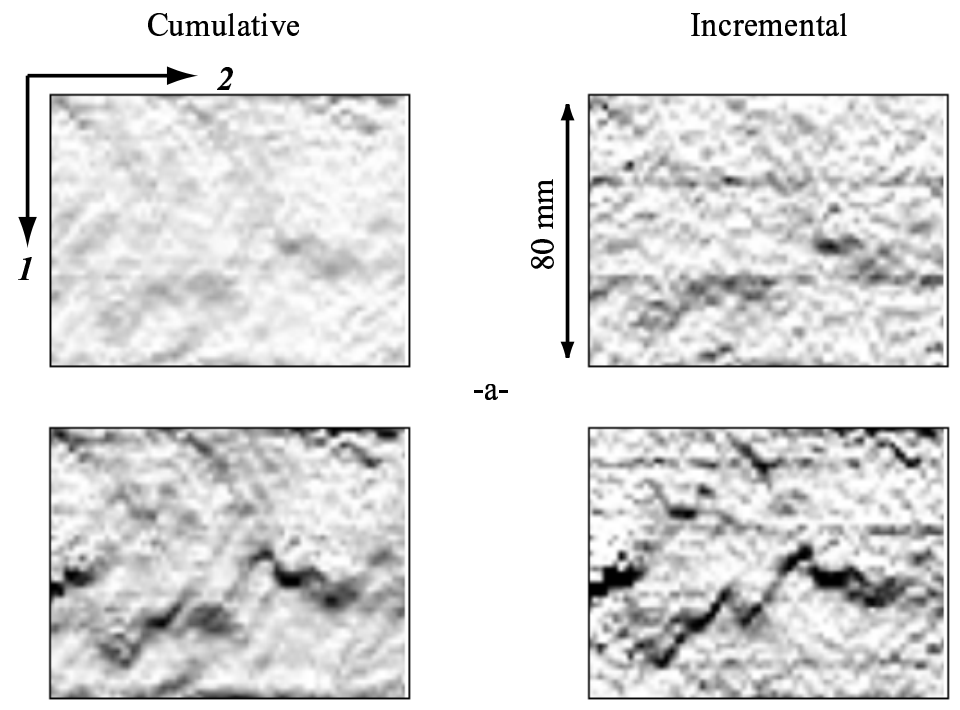

-a-

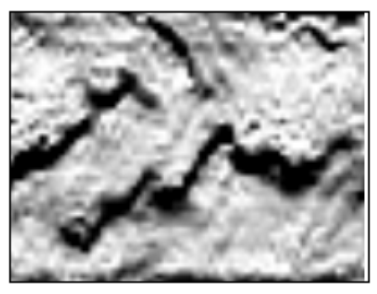

$-b-$
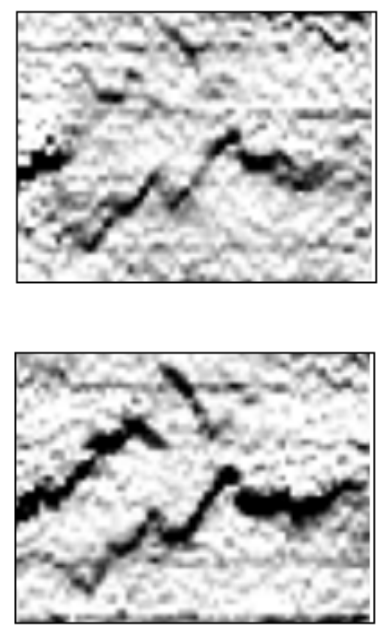

$-\mathrm{c}-$
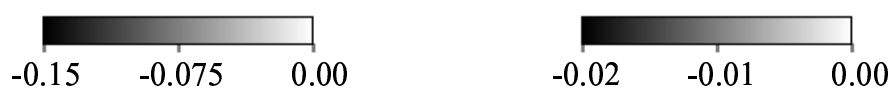

Figure 11: Cumulative and incremental $\epsilon_{11}$-field for different levels of the global strain in a compression test : $\bar{\epsilon}_{11}=-4 \%$ for (a) , $\bar{\epsilon}_{11}=-6.5 \%$ for (b) and $\bar{\epsilon}_{11}=-9 \%$ for (c). Correlation parameters: ZOI size $=32 \times 32$ pixels, $\delta=16$ pixels. Scale: 1 pixel $\leftrightarrow 0.085 \mathrm{~mm}$.

Bergonnier et al. 

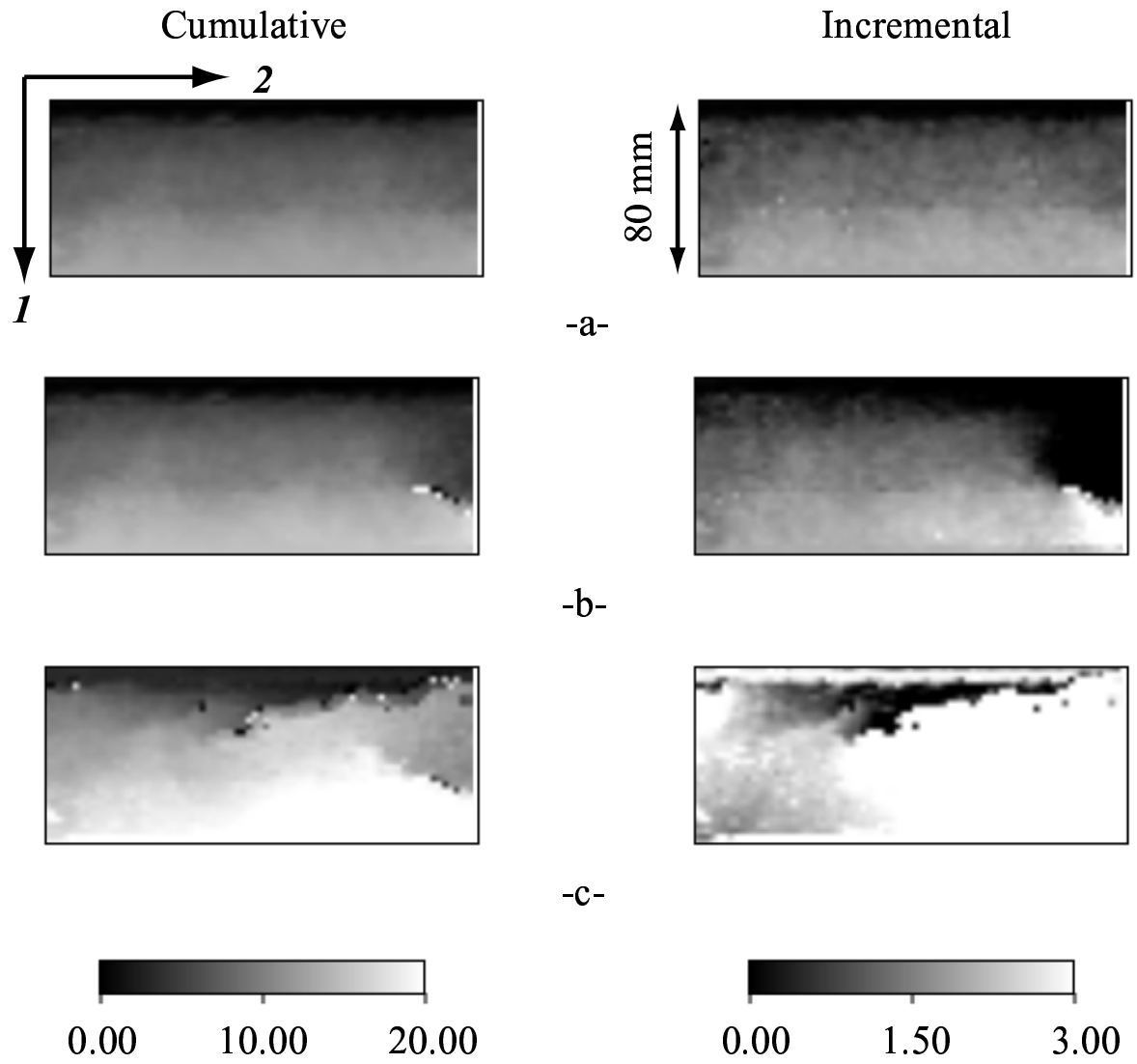

Figure 12: Cumulative and incremental displacement field $u_{1}$ expressed in pixels for different levels of global strain in a tearing test: $\bar{\epsilon}_{11}=4 \%$ for (a), $\bar{\epsilon}_{11}=4.5 \%$ for (b) and $\bar{\epsilon}_{11}=5.5 \%$ for (c). Correlation parameters: ZOI size $=32$ pixels, $\delta=16$ pixels. Scale: 1 pixel $\leftrightarrow 0.16 \mathrm{~mm}$.

Bergonnier et al. 